\title{
Performance limitations of small- format high-speed infrared arrays for active control loops in interferometry and adaptive optics
}

Gert Finger, Roger M. Smith, Serge Menardi, Reinhold J. Dorn, Manfred Meyer, et al.

Gert Finger, Roger M. Smith, Serge Menardi, Reinhold J. Dorn, Manfred Meyer, Leander Mehrgan, Joerg Stegmeier, Alan F. M. Moorwood, "Performance limitations of small-format high-speed infrared arrays for active control loops in interferometry and adaptive optics," Proc. SPIE 5499, Optical and Infrared Detectors for Astronomy, (29 September 2004); doi: $10.1117 / 12.554322$

EDent: SPIE Astronomical Telescopes + Instrumentation, 2004, Glasgow, United Kingdom 


\title{
Performance limitations of small format high speed infrared arrays for active control loops in interferometry and adaptive optics
}

\author{
G. Finger*a, R. Smith ${ }^{\mathrm{b}}$, S. Menardi ${ }^{\mathrm{a}}$, R. J. Dorn ${ }^{\mathrm{a}}$, M. Meyer $^{\mathrm{a}}$, L. Mehrgan ${ }^{\mathrm{a}}$, J. Stegmeier ${ }^{\mathrm{a}}$, \\ A.F.M. Moorwood ${ }^{\mathrm{a}}$ \\ ${ }^{a}$ European Southern Observatory, Karl Schwarzschidstrasse 2 D-85748 Garching, Germany. \\ ${ }^{\mathrm{b}}$ California Institute of Technology, Department of Astronomy, 105-24 Caltech, 1201 East \\ California Blvd, Pasadena CA 91125, USA
}

\begin{abstract}
The detector mounted in the VLTI fringe sensor FINITO is a 256x256 HgCdTe array with a cut-off wavelength of 1.9 micron. The same arrays having cut-off wavelengths of 2.5 micron will be used in the tip tilt sensor IRIS and the PRIMA instrument of the VLT interferometer. The arrays are part of an active control loop with integration times as short as a few hundred microseconds. The fringe tracker FINITO uses only 7 pixels of the array. To take advantage of the four parallel channels of the PICNIC multiplexer, the pixels illuminated in each quadrant are positioned at the same location within the quadrants.
\end{abstract}

A noise analysis of the PICNIC array shows that the main sensitivity limitation of the array is contained in the low frequency part of the noise power spectrum. Similar behaviour has been observed with other infrared arrays. In an effort to optimize the unit cell pixel buffer to achieve high speed and low noise, a prototype multiplexer is being developed at Rockwell for adaptive optics. However, low frequency noise may still be the limiting factor dominating the noise performance of infrared arrays. To overcome this noise barrier, detector architectures have to be envisaged which should allow double correlated sampling on shorter time scales than a full exposure. This might be accomplished by some kind of gate in the IR material which allows charge to be shifted from an integrating well in the infrared pixel to a small sensing node capacitance of the multiplexer unit cell buffer.

Keywords: PICNIC, HgCdTe, fringe tracking, interferometry, adaptive optics, infrared array, detector, high speed

\section{Introduction}

All applications employing real time control loops to either correct wavefront distortions by the atmosphere or optical path length fluctuations due to atmospheric disturbances in optical interferometers require high speed sensors with the lowest possible readout noise. Due to the high bandwidth of the control loop, sensor integration times may be shorter than 1 millisecond. Hence, for fainter guide stars the sensors become read noise limited. At infrared wavelengths currently two sensors are in use, the Hawaii 1 Kx1K array and the Picnic 256x256 array [1]. Since the selected infrared diode arrays are based on $\mathrm{Hg}_{(1-\mathrm{x})} \mathrm{Cd}_{\mathrm{x}} \mathrm{Te}$ the cut-off wavelength can be tailored to the specific application. The wavefront sensor in the Shack-Hartmann AO system of NAOS and the fringe sensor of VINCI use Hawaii1 1Kx1K detectors having cut-off wavelengths of $\lambda_{c}=2.5 \mu \mathrm{m}$. The fringe tracker FINITO is equipped with a PICNIC $256 \times 256 \mathrm{HgCdTe}$ array grown by MBE on a CdZnTe substrate. Its cut-off wavelength is $\lambda_{c}=1.9 \mu \mathrm{m}$. The PRIMA fringe sensors will also be equipped with $\lambda_{c}=2.5 \mu \mathrm{m}$ PICNIC arrays. The noise performance of these devices at high speed is limited. For a simple double correlated clamp the Hawaiil array, which has a pixel size of $18.5 \mu \mathrm{m}$, achieves a readout noise of 10 erms and the PICNIC array because of its larger pixel size of $40 \mu \mathrm{m}$ has a readout noise of 20 to 30 erms.

The performance of real time control loops would benefit enormously by small format high speed infrared sensors with improved readout noise performance. For this reason Caltech contracted with Rockwell, and was joined soon afterwards

*gfinger@eso.org, phone:+49-89-32006256, , +49-89-3200316, fax:+49-89-3202362 
by ESO, to produce a new prototype sensor, which has 7 experimental unit cell designs. Each unit cell design will be evaluated and compared. The most promising design will be used to fabricate a new uniform small format $256 \times 256 \mathrm{AO}$ sensor [2].

\section{Fringe tracker FINITO}

From the beginning the VLT was designed to operate as an interferometer. The light collected by the individual 8 meter telescope is corrected by a wavefront curvature adaptive optics system and transferred by the delay line system to the beam combination laboratory. Since the atmospheric turbulence introduces an optical path difference (OPD) between the beams from different telescopes, the integration time of VLTI instruments is limited to a few milliseconds because of the short atmospheric coherence time. If the OPD can be actively controlled and stabilized, instruments using the combined focus can perform long integrations which results in a sensitivity gain of several magnitudes.

Since the bandwidth of the VLTI OPD control loop should be up to $40 \mathrm{~Hz}$ to allow effective compensation of OPD fluctuations and because of the phase detection scheme used in FINITO, the detector has to be read out with frame rates up to $4 \mathrm{KHz}$.

The layout of the fringe sensor FINITO is shown in Figure 1. The dichroics feed the $\mathrm{H}$ band of the beams from three telescopes into FINITO []. The beams are combined with the metrology laser which has a wavelength of 1310nm and is used to control the OPD modulators in closed loop. The triangle like OPD modulation is accomplished by piezoelectric cylinders which stretch the fibers wrapped around the cylinders. Polarisers split the beams into photometric and interferometric beams. The interferometric beams are combined by a beam-splitter group which generates 4 combined beams. The 3 photometric and 4 combined beams are routed to the cryostat by single-mode fibers, which are imaged onto the detector. Coherently combined pairs of beams are both measured in transmission and reflection with a corresponding phase shift of $\pi$. The re-imaging optics is arranged such, that the 7 fiber outputs are imaged on two groups of 4 pixels having the same position in each of the four quadrant of the PICNIC array. By doing so 4 pixel can be read out in parallel. The corresponding gain in bandwidth allows more nondestructive readouts for the same analog bandwidth of the detector, which further reduces the readout noise.

The Picnic detector set-up is shown in Figure 2. The internal bus of the multiplexer is accessed directly bypassing the on-chip source follower. Cryogenic CMOS preamplifers for all 4 video channels are located on the backside of the fanout board. The symmetric amplifier design provides a robust true differential signal chain which shows a high degree of immunity to noise pick-up.

Even though the nominal readout noise of the PICNIC array is higher than the readout noise of the Hawaii1 array, due to the larger pixel size of $40 \mu \mathrm{m}$ only one pixel has to be read per fiber. As much as $70 \%$ of the total signal can be concentrated on a single pixel with the PICNIC array, whereas less than $50 \%$ can be achieved with the smaller pixels of the Hawaiil array. Even if perfect optics illuminates only a single pixel the signal spreads to the neighboring pixels by lateral charge diffusion and at least $2 \times 2$ pixels have to be read with the Hawaii 1 array, which ruins the better noise performance of the Hawaii1. For this reason and because of its lower price the PICNIC array was chosen.

The infrared array is read out synchronously with the OPD modulation. The residual OPD is estimated by the ABCD algorithm. The intensity of each fiber image is read after each quarter-wave of modulation as shown in Figure 5. Since both transmitted and reflected beam combinations with the corresponding phase shifts of $\pi$ are measured simultaneously only two reads of the 4 interferometric outputs are required to obtain a full set of ABCD samples required to estimate the OPD. If only two telescope beams are combined, two interferometric and two photometric outputs have to be read. If the fibers are positioned properly with each fiber image located in a different quadrant on the same pixel, the array need not be clocked at all. The same pixel of each quadrant is permanently connected to the output and can be sampled continuously without any overhead due to clocking between two pixels, which is only necessary for fringe tracking of three telescope beams. 
Both the OPD modulator and the infrared detector readout are triggered by pulses issued by a time reference module (TIM). In double correlated readout mode a TIM pulse triggers a read-reset-read sequence. The first readout corresponds to the end of the detector integration ramp. The second read, which is performed after the reset, corresponds to the start of the next integration ramp. In Fowler mode the single read is replaced by $n$ reads and the average is used to improve the readout noise. The integration time is defined by the time interval between two TIM pulses. To match the control loop bandwidth of the VLTI typical detector integration times range between $125 \mu$ s to a few ms.

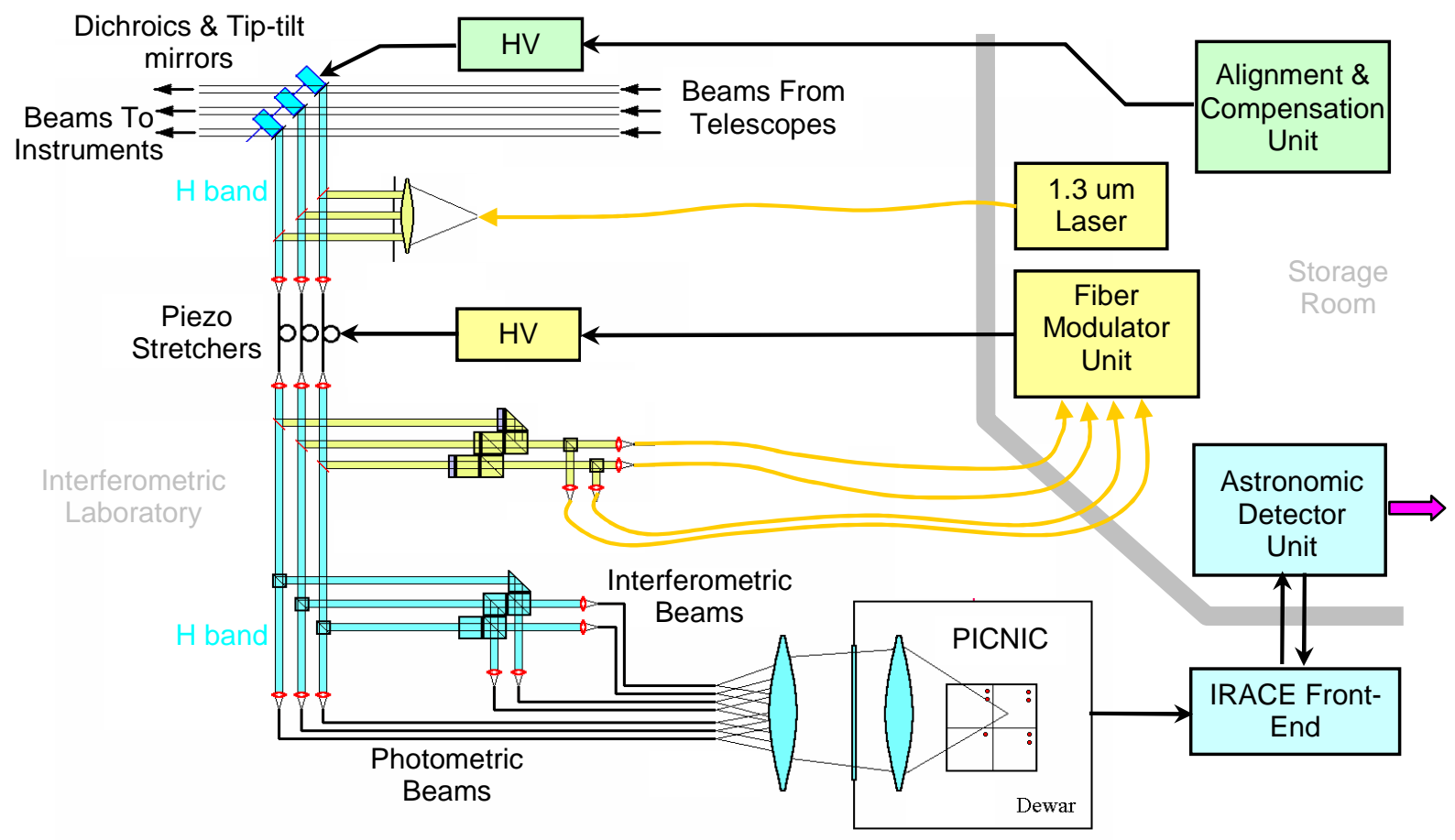

Figure 1 Layout of fringe sensor FINITO with piezoelectric OPD modulators, laser metrology system, beam combiners and infrared sensor. The infrared detector is a 256x256 element PICNIC array grown by MBE on CdZnTe and has a cut-off wavelength of $\lambda_{\mathrm{c}}=1.9$ $\mu \mathrm{m}$.

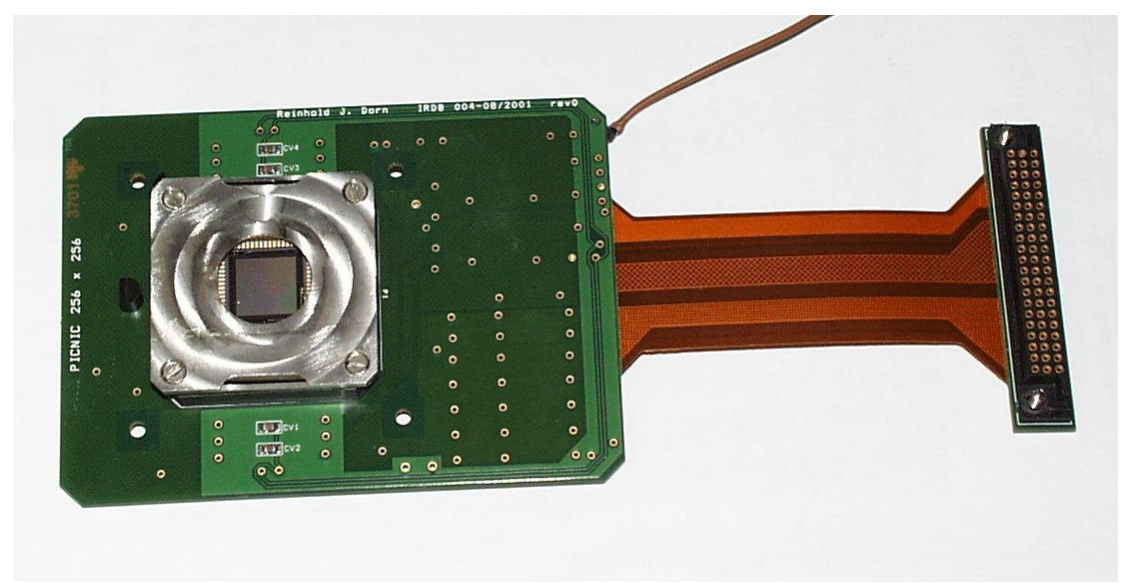

Figure 2 Picnic 256x256 HgCdTe MBE array as used in the FINITO fringe sensor unit. On the backside of the fan-out board cryogenic CMOS preamplifiers for 4 video channels are located. 

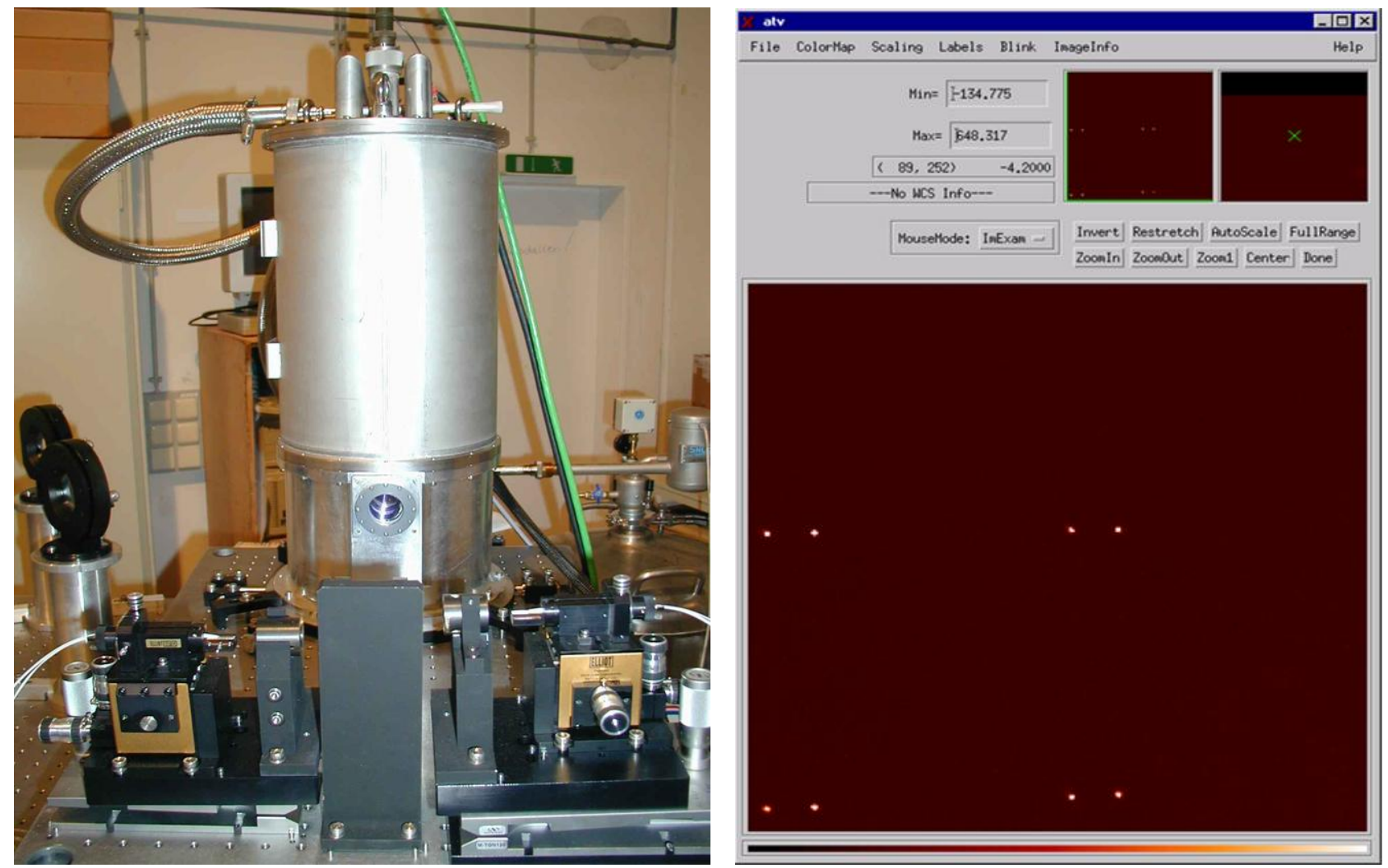

Figure 3 Left: FINITO detector dewar with fiber re-imaging optics. Right: Infrared image of fiber outputs.

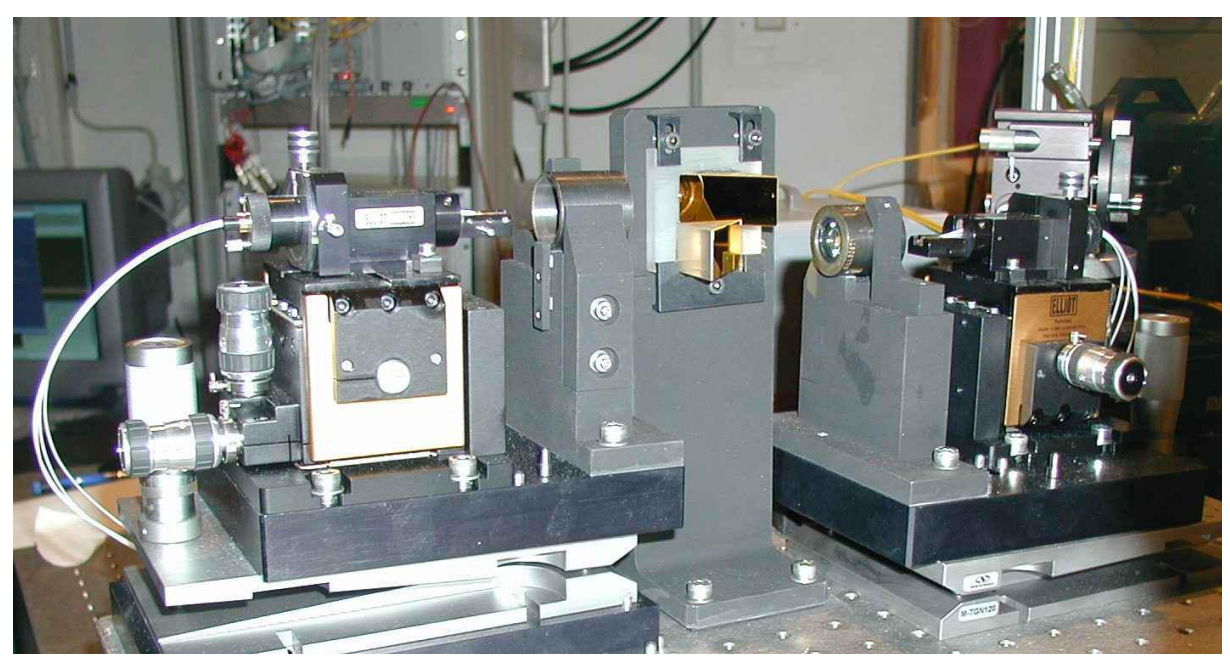

Figure 4 FINITO fiber re-imaging collimator optics. The two mirrors image two groups of 4 pixels of the 7 fiber outputs

(3 photometric and 4 interferometric outputs, one spare) onto the same pixels in each of the four quadrant of the PICNIC array. 


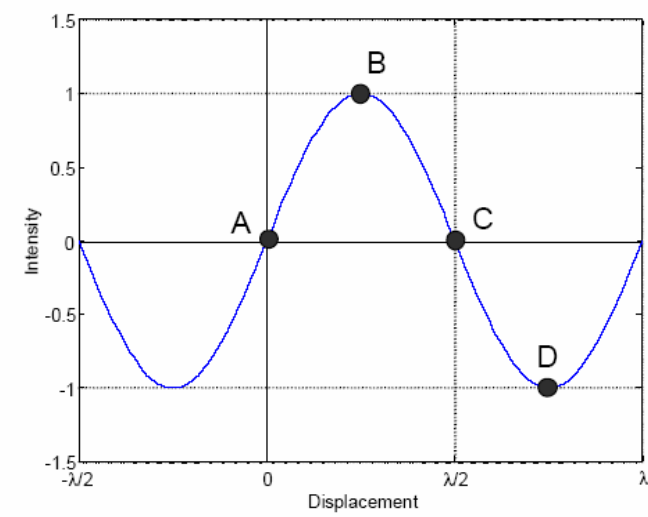

$$
\overline{O P D}=\frac{\lambda_{0}}{2 \pi} \arctan \left(\frac{A-C}{B-D}\right)
$$

Figure $5 \mathrm{ABCD}$ algorithm to estimate residual OPD by sampling an interferometric fiber output 4 times per wavelength.

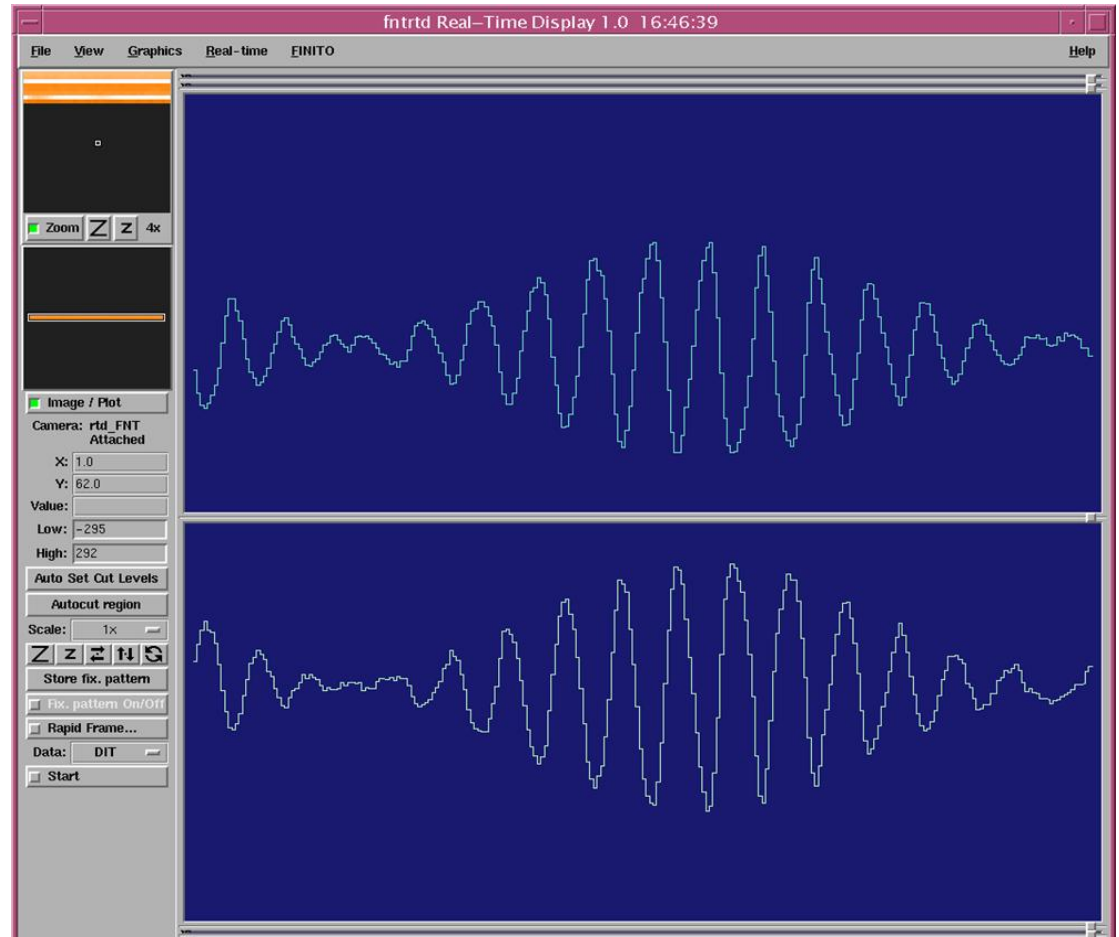

Figure 6 Interferogram as seen on the real time display. Top and bottom interferograms are phase shifted by $\pi$.

\section{Noise analysis of PICNIC array}

Since as few as 2 pixels per quadrant for the combination of three telescopes and only one pixel per quadrant for the combination of two telescopes have to be read out with the PICNIC array, it was hoped that the readout noise can be reduced to a few electrons by multiple sampling techniques. By Fowler sampling the white noise is reduce by 
1/SQRT(n) with $\mathrm{n}$ being the number of nondestructive readouts. To examine the noise behavior the output was sampled every $500 \mathrm{~ns}$. The raw signal is shown in Figure 7. If the output is smoothed by a running average of 200 samples as shown in Figure 8 it becomes evident that the low frequency noise content is dominant and the noise reduction does not follow the square root of $n$ law.

The power spectral density of the signal is shown in Figure 9. The spectra of the raw spectrum multiplied with the transfer functions of double correlated sampling for sampling intervals of $1 \mathrm{~ms}$ and $250 \mu$ s are also shown. For an integration time of $250 \mu$ s the low frequencies are substantially attenuated. The readout noise, which is the power spectral density PSD integrated over all frequencies, is reduced from 40 erms to 20 erms when the integration time is reduced from $2 \mathrm{~ms}$ to $250 \mu \mathrm{s}$ as shown in Figure 10 which displays the readout noise for 2 different pixels as function of integration time.

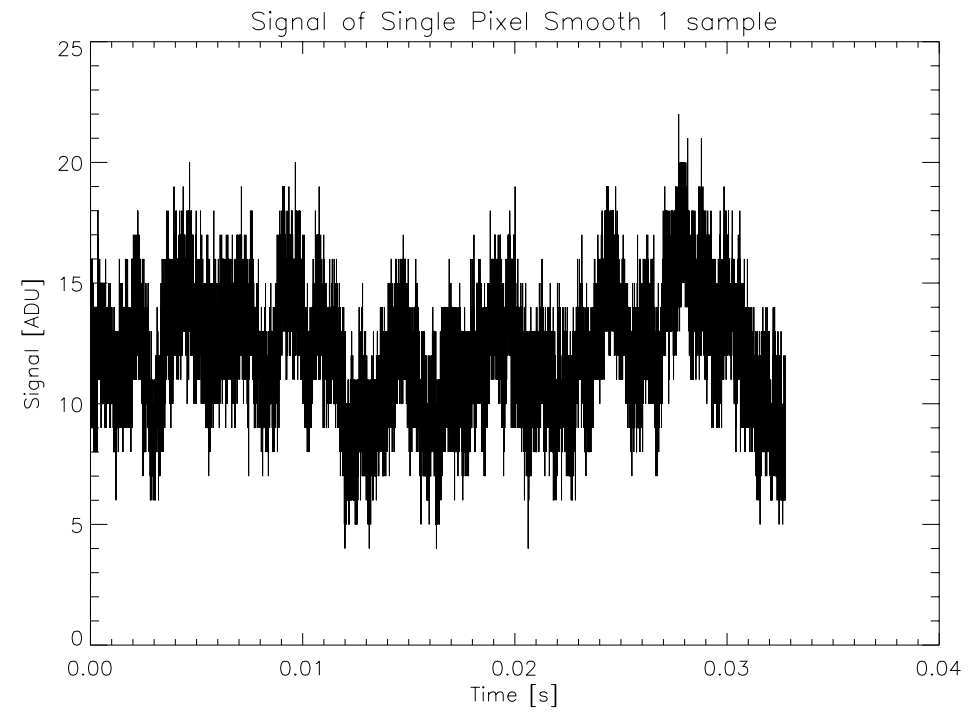

Figure 7 Raw output of PINIC array. Sampling frequency $500 \mathrm{~ns}$.

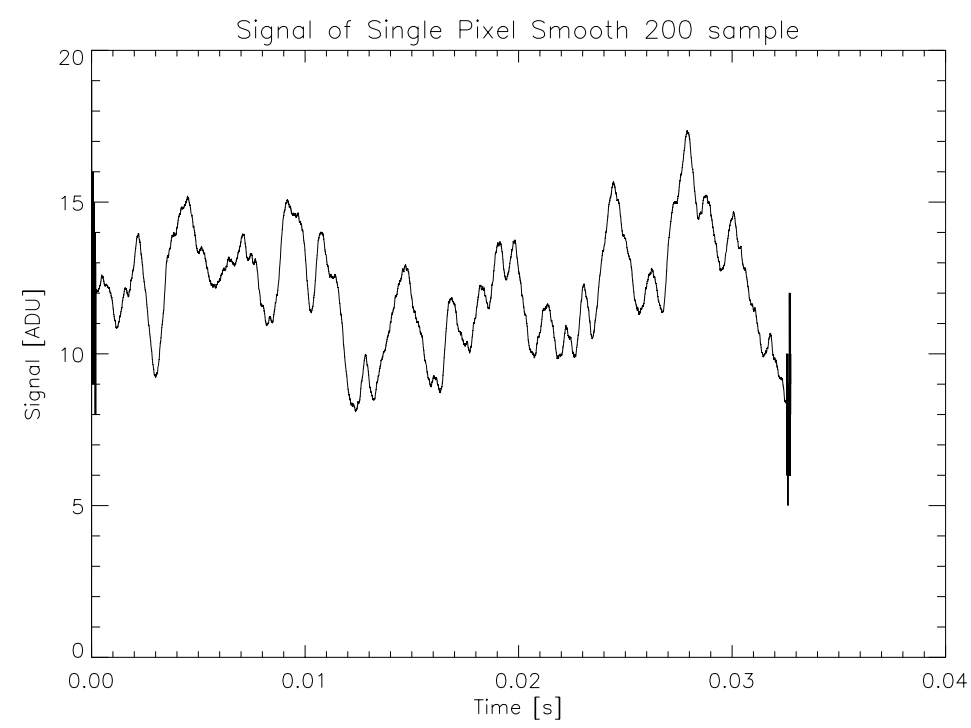

Figure 8 Output of PICNIC array smoothed with average of 200 samples. Low frequency noise is dominant 


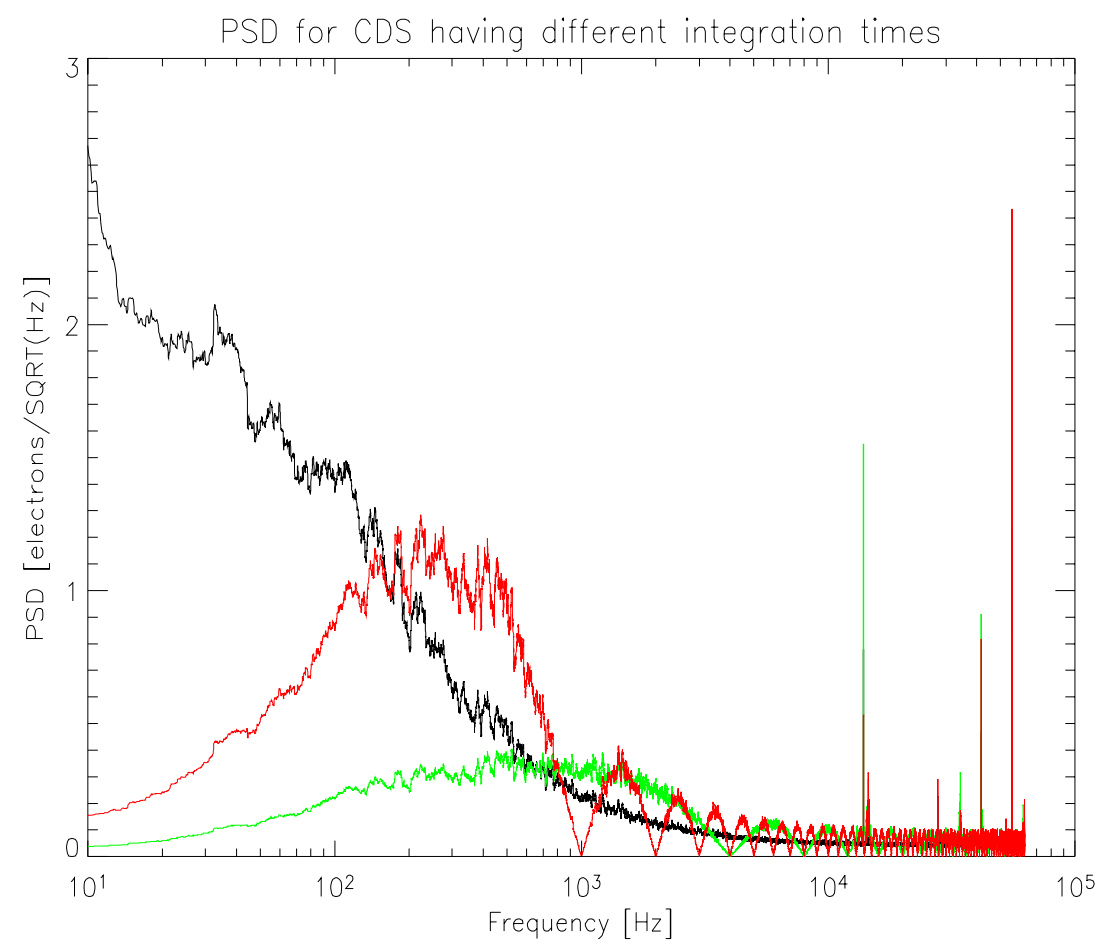

Figure 9 Power spectral density of PICNIC array. Raw spectrum and spectra multiplied with transfer function of double correlated clamp for sampling intervals of $1 \mathrm{~ms}$ and $250 \mu \mathrm{s}$.

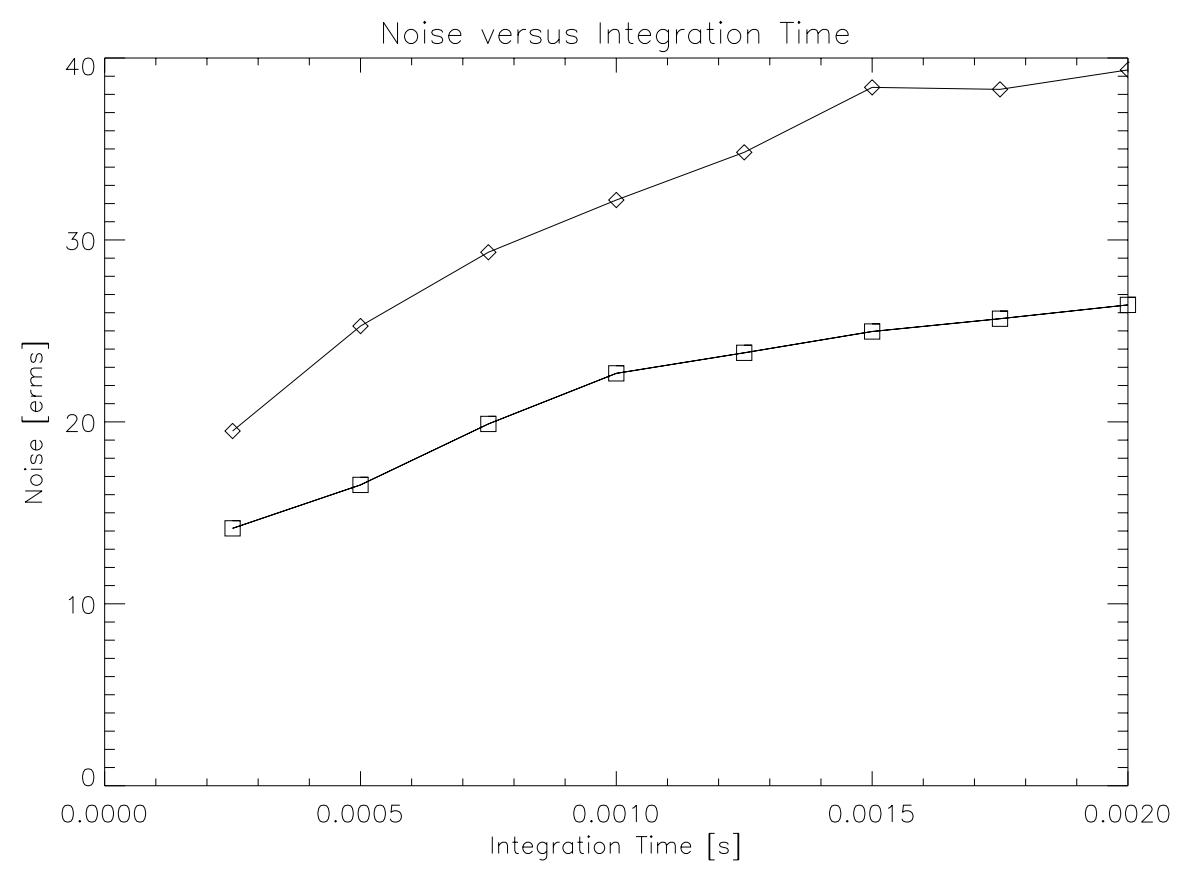

Figure 10 Measured readout noise of PICNIC array versus exposure time for two different pixels. 
In Figure 11 the cumulative power spectral density is shown, which is the power spectral density integrated from zero to frequency $\mathrm{f}$ and gives the noise contribution in electrons rms for the frequency interval 0 to $\mathrm{f}$. The upper curve is the cumulative PSD for normal operation of the detector in capacitive discharge mode with the reset switch open. The lower curve was measured with the reset switch permanently closed. For exposure times of many seconds the CDS process only removes the noise at extremely low frequencies, so the total noise of 32 erms is composed of a major contribution of 10 erms originating from frequencies between 0 and $1 \mathrm{~Hz}, 18$ erms are contributed from frequencies between $1 \mathrm{~Hz}$ and $1 \mathrm{KHz}$ and higher frequencies generate as little as 4 erms. If the reset switch is permanently closed, as represented by the lower curve in Figure 11, the cumulative PSD looks completely different. The low frequency contribution below $1 \mathrm{~Hz}$ is only 1 erms and the distribution resembles a white noise spectrum. From the comparison of the two curves in Figure 11 it is concluded that the readout noise is not dominated by noise of the unit cell source follower, the multiplexer or the rest of the acquisition chain. The main noise source is located ahead of the gate of the unit cell source follower. The noise is most probably generated by capacitive coupling of low frequency noise sources on the integrating node of the detector or it is generated by the infrared diode itself.

The spectral noise distribution is concentrated at frequencies below $1 \mathrm{KHz}$. Elaborate multiplexer designs which perform well for high bandwidths and fast read speeds, do not overcome the problem of low frequency noise suppression. One way to eliminate the low frequency noise is a double correlated sampling scheme, in which the time interval between the two samples is not defined by the exposure time as it is the case for infrared detectors operating in capacitive discharge mode. If the sampling interval is defined only by the analog bandwidth of the detector output, the sampling interval can be as short as $1 \mu \mathrm{s}$ and low noise frequencies are eliminated by the filter function of the double correlated clamp. The charge sensing node is reset and the voltage on sensing node is measured. Then the signal charge is transferred onto the sensing node and the voltage on the sensing node is measured again. The difference is the double correlated clamp. This is exactly how the output stage of a CCD eliminates low frequency noise.

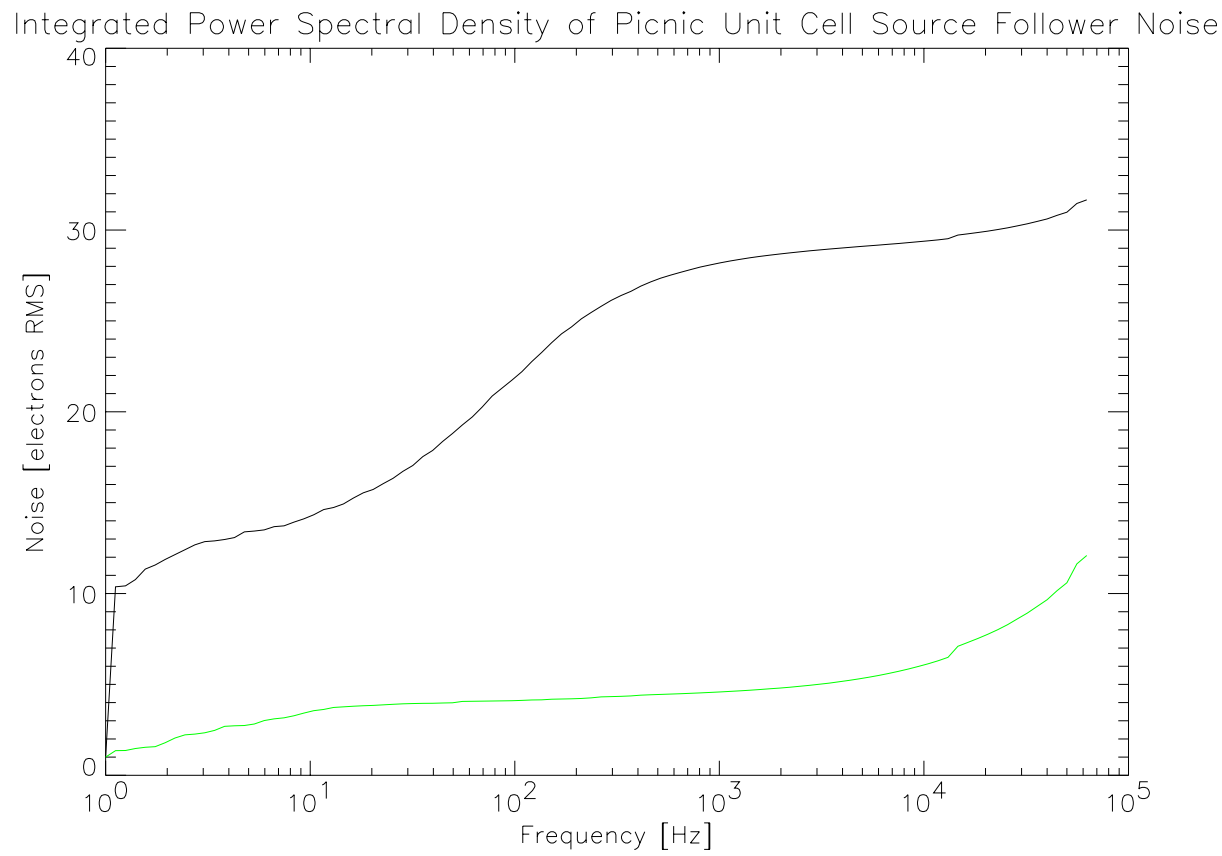

Figure 11 Cumulative Power spectral density function. Upper curve: normal operation, capacitive discharge mode. Lower curve: reset switch permanently closed. 


\section{Prototype AO multiplexer}

To improve the performance of the FINITO fringe tracker and the infrared wavefront sensor in NAOS both Caltech and ESO have contracted with Rockwell to develop a new prototype sensor, the Calico chip [6]. It has 7 different unit cell designs. Each unit cell design will soon be tested and the most promising one will be chosen to manufacture a uniform small format $256 \times 256 \mathrm{AO}$ sensor. The underlying idea is that by placing the signal processing circuitry under each pixel it is possible to filter the noise prior to multiplexing. The whole exposure time is then available for limiting noise bandwidth rather than just the pixel time. The gain at the pixel assures that the input noise remains dominant even at higher noise bandwidth required when addressing the pixels more rapidly.

The basic parameters of the Calico chip are summarized in Table 1. The readout noise depends on the unit cell design and the type of sensor hybridized to the multiplexer. For HgCdTe infrared diodes which have a capacity of $\sim 30 \mathrm{fF}$, the expected readout noise at 800 frames/sec is $\sim 7 \mathrm{erms}$ and for silicon pin diode arrays which have a capacity of $\sim 5 \mathrm{fF}$ the readout noise is predicted to be $\sim 3.5$ erms. Some of the unit cell designs are not linear by design. The most promising design is a unit cell with capacitive transimpedance amplifiers. The floor plan of the multiplexer is shown in Figure 12. Each unit cell design consists of a strip of 16x128 pixels and can be operated with 8 parallel outputs.

Table 1 Specifications for Calico AO multiplexer

\begin{tabular}{|l|l|}
\hline Parameter & Specification \\
\hline Wavelength range & $0.8-2.3 \mu \mathrm{m}$ \\
\hline Material & MBE grown HgCdTe on CdZnTe \\
\hline Format & $128 \times 128$ \\
\hline Pixel pitch & $40 \mu \mathrm{m}$ \\
\hline Number of parallel outputs & 8 \\
\hline Pixel clock & $286 \mathrm{~ns}$ \\
\hline Frame rate & $2.71 \mathrm{KHz}$ \\
\hline Readout Noise & $\begin{array}{l}7 \text { erms @ 800 frames/s } \\
\text { depends on unit cell design }\end{array}$ \\
\hline
\end{tabular}
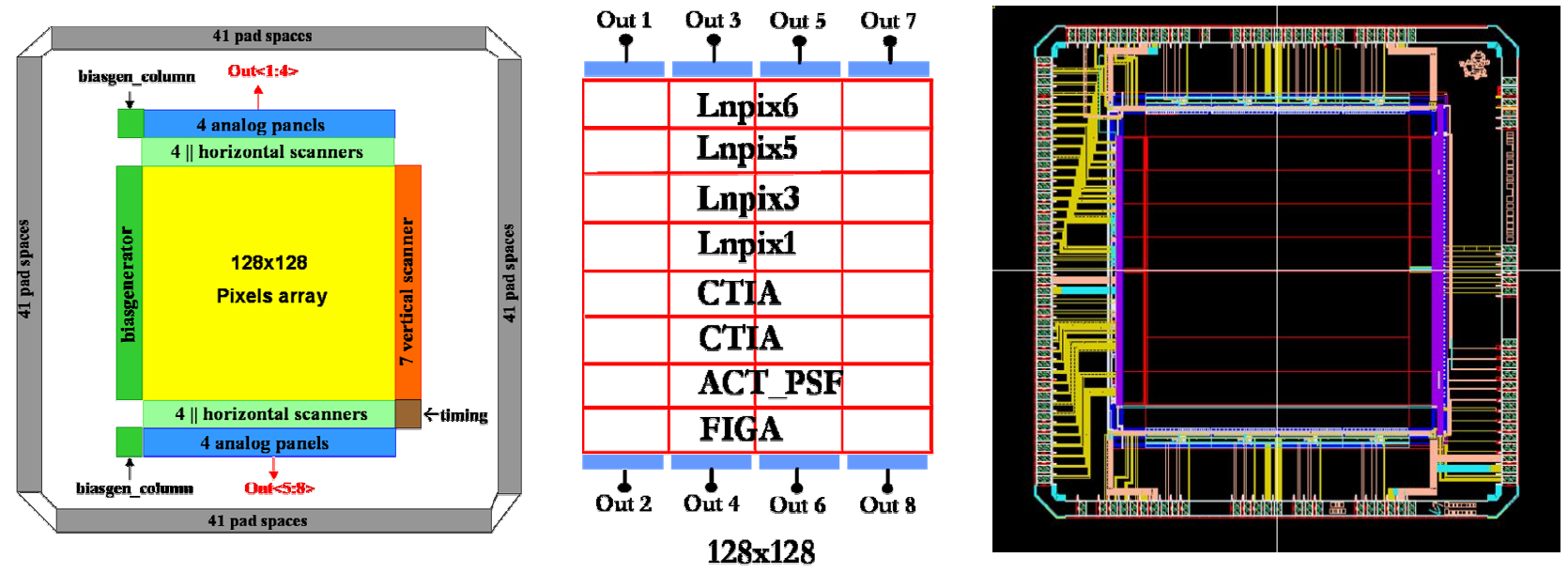

Figure 12 AO prototype multiplexer CALICO: Left: floor plan. Middle: layout of unit cell designs. Right: AO mux top level layout. 


\section{Alternative pixel architectures}

The band limiting provided by the Calico Mux can filter out the high frequency noise components but cannot overcome the problem of low frequency noise suppression. The Calico mux is being hybridized to both Si PIN diode arrays and $2.5 \mu \mathrm{m} \mathrm{HgCdTe}$. If either of these materials has a spectral noise distribution concentrated at frequencies below $1 \mathrm{KHz}$, as is the case for the $1.9 \mu \mathrm{m}$ cut-off PICNIC array described in Figure 11, then the gains provided by the Calico mux may be limited.

One way to eliminate the low frequency noise is a double correlated sampling scheme, in which the time interval between the two samples is not defined by the exposure time as is the case for infrared detectors operating in capacitive discharge mode. If the signal charge can be transferred to the sense node soon after the sense node is reset, the low noise frequencies are eliminated by the filter function of the double correlated clamp as shown in Figure 9. Two readout architectures are proposed to achieve this.

\subsection{Infrared diode array with silicon $\mathrm{CCD}$ and direct injection input stage}

HgCdTe diode arrays have been built two decades ago, which were hybridized onto CCD type unit cells as shown on the right side of Figure 13. The input to the CCD is a direct injection FET. These devices suffered from threshold nonuniformities of the direct input injection stages and poor charge injection efficiency [7]. The input impedance of the direct injection stage is inversely proportional to the input current and increases for low flux applications. However, in the past two decades both the threshold uniformity of MOS FET's has improved and the impedance of infrared detectors has increased dramatically. In view of these improvements the direct injection may become attractive for AO applications which do not look at extremely faint sources and are less affected by low injection efficiency. Furthermore, CCD readouts can be provided with an extension of the serial register which is operated with high clock voltages and multiplies charge by the avalanche effect. This L3CCD type serial register adds gain and the CCD can be read out in the photon counting mode [8].

\subsection{Charge transfer pixel architecture}

If a "1-pixel CCD" could be manufactured in IR detector materials then the charge could be transferred rapidly from the detecting area to a small sense node in the corner of each pixel as shown on the left side of Figure 13. This concept has already been developed by J.Janesick in Silicon[5]. On-pixel processing electronics similar to the Calico Mux would perform the correlated double sampling.

Such a design would boost the signal relative to the input transistor noise by moving the signal noiselessly to a low capacitance sense node. This sense would still be in a potentially noisy detector material but detector noise might be expected to scale down by the square root of the area. Since the charge would be dumped on the sense node at the end of the exposure, the CDS time could be optimized for the best compromise between rejecting 1/f noise and white noise, instead of being given by the exposure or pixel times. The realization of this type of readout for infrared hybrid arrays depends on the availability of metal-insulator-semiconductor structures for III-V or IV-VI compounds. 

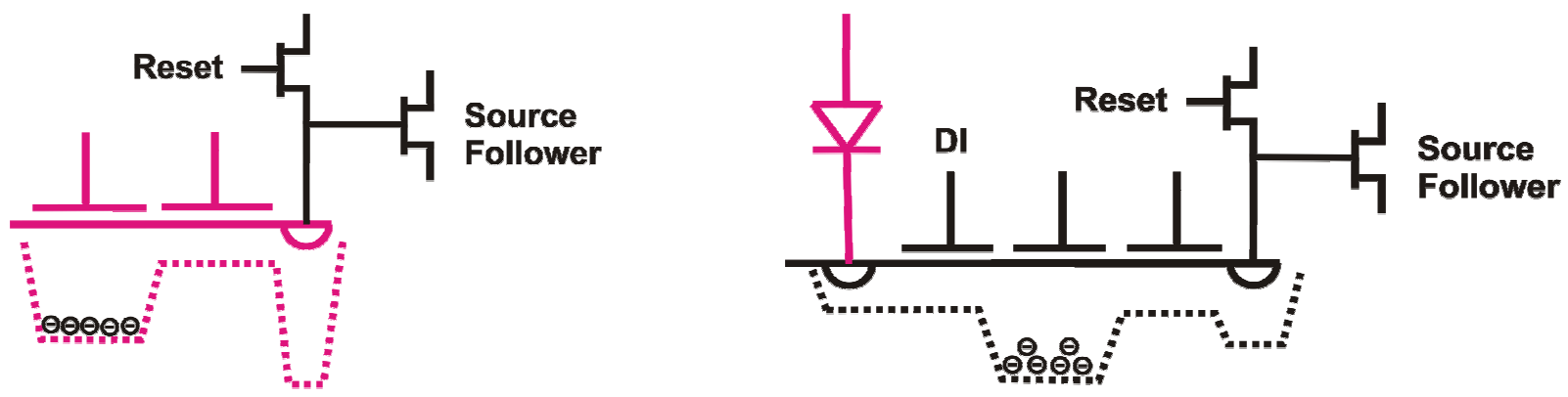

Figure 13 Left: Infrared CCD type unit cell design with Si CMOS source follower per pixel. Right: Infrared diode array with direct injection gate to Si CCD unit cell and source follower per pixel.

\section{Conclusions}

At present the Hawaii $1 \mathrm{Kx} 1 \mathrm{~K}$ and the PICNIC 256x256 HgCdTe arrays are used for infrared wavefront sensing and fringe tracking. An MBE grown PICNIC array, which has a cut-off wavelength of $1.9 \mu \mathrm{m}$, has successfully been installed in the fringe tracker FINITO at the VLTI. First fringes have been obtained and the OPD control loop closed efficiently. Thanks to the re-imaging optics, the 7 fiber outputs of the fringe sensor are imaged on the same pixels of each quadrant, which allows exploiting the multiplex advantage of the 4 parallel outputs of the PICNIC array.

The readout noise of these detectors limits the performance of adaptive optics systems and real time control loops used for interferometry. A detailed analysis of an MBE grown PICNIC array shows, that the main noise contribution is concentrated at low frequencies below $1 \mathrm{KHz}$ and is not originating within the multiplexer or the rest of the acquisition chain. The noise is generated in or coupled onto the integrating node. A scheme which allows reducing the sampling interval of a double correlated clamp to time scales shorter than the $1 / \mathrm{f}$ corner frequency in the noise spectrum while not affecting the exposure time will effectively suppress the low frequency noise. We have suggested several possible methods for achieving this.

\section{REFERENCES}

1. E. Gendron, F.Lacombe, D. Rouan, J.Charton, B. Lefort, C. Marlot, G. Michet, G. Nicol, S. Pau, V. D. Phan, B. Talureau, J.L.Lizon, N.Hubin, "NAOS Infrared wave-front sensor design and performance", Proceedings SPIE 4839, pp. 195-205, 2003.

2. John Blackwell, Rockwell Scientific, private communication.

3. Gai, M.; Bonino, D.; Corcione, L.; Delage, L.; Gardiol, D.; Gennai, A.; Lattanzi, M. G.; Loreggia, D.; Massone, G.; Menardi, S.; Reynaud, F., Fringe tracking for VLTI and LBT”, Memorie della Societa Astronomica Italiana, v.74, p.130, 2003.

4. M. Gai et al., "The VLTI Fringe Sensors: FINITO and PRIMA FSU", Proceedings SPIE 5491, to be published.

5. Janesic, J.,"Charge coupled CMOS and hybrid detector arrays", Proceedings SPIE 5167, pp. 1-18, 2004.

6. A. Joshi, A. Konenenko, J. Stevens, "Ultralow Noise high frame rate ROIC for infrared focal plane arrays", Proceedings SPIE 5499, to be published.

7. G. Finger, M.Meyer, A.F.M. Moorwood, "Test results with Mullard CMT-CCD hybrid focal plane arrays", Proceedings SPIE 865, pp 94-101, 1987.

8. A. Basden, C. Haniff, C. Mackay, "L3CCD's: Fast photon counting for optical interferometry", Workshop on Scientific Detectors for Astronomy, pp. 593-597, Kluwer, 2004. 